\title{
Multiple facets of response to fungicides - the influence of azole treatment on expression of key mycotoxin biosynthetic genes and candidate resistance factors in the control of resistant Fusarium strains
}

\author{
D. Popiel • A. Dawidziuk • G. Koczyk • A. Mackowiak • \\ K. Marcinkowska \\ Accepted: 23 August 2016/Published online: 31 August 2016 \\ (C) The Author(s) 2016. This article is published with open access at Springerlink.com
}

\begin{abstract}
The spread of fungicide resistant and/or tolerant phytopathogenic fungi is an important factor affecting crop protection. However, the mechanisms of fungal response to fungicide application are not entirely characterised. In particular, the contribution of previously known resistance factors and the final influence of fungicide treatments on metabolism of surviving mycelia (e.g. mycotoxin increased release and biosynthesis potentially causing contamination of the crops) merit investigation, in order to improve future molecular diagnostics of fungicide resistant strains. The performed experiments have shown distinct expression changes for homologs of a known fungicide resistance factor Flr1 (yeast; DHA1 family of major facilitator superfamily transporters) after azole application in cultured fusaria. Two distantly related homologs of that gene were selected, based on the unsupervised clustering and phylogenetic analysis of transporter sequences. One of these (FGSG_02865), was found to occur across the Fusarium sambucinum complex (F. graminearum,
\end{abstract}

Electronic supplementary material The online version of this article (doi:10.1007/s10658-016-1042-3) contains supplementary material, which is available to authorized users.

D. Popiel $(\bowtie) \cdot$ A. Dawidziuk $\cdot$ G. Koczyk •

A. Mackowiak $\cdot$ K. Marcinkowska

Institute of Plant Genetics Polish Academy of Sciences,

Strzeszyńska 34, 60-479 Poznan, Poland

e-mail: dpop@igr.poznan.pl

A. Mackowiak $\cdot$ K. Marcinkowska

Faculty of Agronomy and Bioengineering, University of Life

Sciences, Wojska Polskiego 26, 60-637 Poznan, Poland
F. culmorum, F. cerealis) and was upregulated starting $24 \mathrm{~h}$ after fungicide treatments. This delayed response may point to possible involvement of DHA1 antiporters in a generalised response to stress resulting from fungicide treatment. Additional expression profiling was conducted for the mycotoxin biosynthetic genes (trichothecene and zearalenone gene clusters) in strains of Fusarium sambucinum complex cereal pathogens. The expression changes, when subjected to treatment with the fungicides (flusilazole, carbendazim), show that even an effective treatment (in this study, the benzimidazole fungicide carbendazim) applied to the grown mycelium, can result in enhanced activation of mycotoxin biosynthetic genes in fungal cells which survive the treatment. Our results suggest that increased mycotoxin contamination can be strongly influenced not only by the amount or the type of antifungal compound, but also the timing of fungicide exposition (stage of infection).

Keywords Mycotoxins $\cdot$ Efflux pumps $\cdot$ Fungicide resistance $\cdot$ Mycotoxins $\cdot$ Fusarium

\section{Introduction}

One of the most important fungi occurring in agricultural environment are the members of the Fusarium genus. These fungi are cosmopolitan plant pathogens, occurring throughout the world and are able to infect in a short time both generative and vegetative parts of their hosts. Fusarium strains are the major cereal crop pathogens responsible for significant losses in crop yield due 
to both diminished biomass and mycotoxins accumulated in the infiltrated parts, e.g. zearalenone, fumonisins, trichothecenes and their derivatives (D'Mello et al. 1999). The plant pathogenic fusaria are present in the soil, air and on crops of all continents with some of the common species being: Fusarium culmorum, F. graminearum, $F$. avenaceum, $F$. verticillioides and $F$. proliferatum. The survival of fungal plant pathogens, such as Fusarium sp., in cultivated fields subjected to the chemical treatments, depends mainly on the development of multiple adaptive mechanisms against different antifungal agents - both natural (such as antifungal mycotoxins) and synthetic fungicides.

In the latter case, the mechanisms of response to demethylation-inhibiting (DMI) fungicides relate both to qualitative factors such as the absence or presence of a sensitive target site, as well as quantitative factors such as uptake, transport, storage and metabolism (Cools et al. 2013; Deising et al. 2008). The genetic determinants of resistance/tolerance can act in a synergistic manner, such as overlapping transporter activity (Rogers et al. 2001). The role of multiple membrane transporters in azole resistance has been particularly well characterised for human pathogens (Costa et al. 2014), however there is less knowledge on the involvement of transporters in plant pathogen resistance to DMI's, with inquiries showing complex responses in regards to treatment with different antifungal/toxic substances (e.g. Thurau et al. 2013). Recently, transcriptome screening for genes highly expressed during azole treatment (Becher et al. 2011; Liu et al. 2010) has allowed the researchers to verify several contributing transporters in F. graminearum (Abou Ammar et al. 2013). Still, translational research aiming to characterise homologs of known resistance factors from baker's yeast and human pathogenic species (such as Candida albicans or Aspergillus fumigatus) in the context of plant pathogenic fungi has been lacking (Price et al. 2015). The major facilitator superfamily (MFS) proteins constitute one of the largest transporter superfamilies, represented throughout all three domains of life (Archaea, Eubacteria and Eukaryota; Saier 1999). This group of secondary efflux pumps is demonstrably involved in the uniport, symport, or antiport of various compounds, coupled to changes in chemiosmotic ion gradients. Of the various subgroups, the DHA1 antiporter family contains several high-impact fungicide resistance factors present in yeasts. These were experimentally characterised in both nonpathogenic (Flr1 - fluconasol resistance from the baker's yeast Saccharomyces cerevisiae; Alarco et al. 1997, caf5 caffeine resistance protein from the fission yeast Schizosaccharomyces pombe; Benko et al. 2004, CYHR - cycloheximide resistance protein from Scheffersomyces stipitis; Sasnauskas et al. 1992) and human pathogenic yeast species (MDR1 - benomyl/ methotrexate transporter from pathogenic strains of Candida albicans; Fling et al. 1991).

One additional problem with quantitative resistance to fungicides (e.g. MDR) is that a substantial part of the susceptible mycelium could survive the chemical treatments and potentially carry out the biosynthesis and efflux of toxic secondary metabolites (Simpson et al. 2001). Therefore an additional challenge posed by the spread of increased resistance to fungicides, is that of the possible role of surviving pathogen (e.g. enhanced mycotoxin contamination after fungicide treatment) even in a partially susceptible population (Schmidt-Heydt et al. 2013).

Notably, as a rule, the mycotoxin biosynthetic genes are not constitutively expressed, but instead are induced in certain stress-related conditions and/or growth phases of the fungus. In context of the fungicidal treatments, there is a lot of evidence that non-lethal amounts of fungicides, could lead to increased mycotoxin biosynthesis (as evidenced in gene expression patterns). According to the past results of Ochiai et al. (2007), suboptimal doses of triazole fungicides can transcriptionally activate the Tri5 gene (trichothecene synthase) in Fusarium graminearum. Similar results were obtained in the experiments investigating the influence of sodium propionate and potassium adsorbate on the expression of the Penicillium verrucosum ochratoxin polyketide synthase gene (Schmidt-Heydt et al. 2007). The aim of the present work was to: (i) characterise whether the putative resistance mechanisms from DHA1 family (e.g. expressed Flrl homologs) are shared by distantly related fungi from Fusarium genus, and (ii) to what extent those genes are involved in reaction to fungicide treatments. Additionally, the expression profiling allowed us to address the question if suboptimal doses of fungicides can cause distinct changes in the production of mycotoxins. The transcriptional response was studied for key mycotoxin biosynthetic genes involved in both trichothecene (Tri5 - trichodiene synthase, Tri6 - trichothecene cluster transcription factor, Tri12 trichothecene efflux transporter; Desjardins and 
Proctor 2007) and zearalenone biosynthesis (Zea2 zearalenone polyketide synthase, Zeb2 - zearalenone transcription factor and Zral - putative efflux pump; Lee et al. 2011).

\section{Materials and methods}

Samples collection and identification

The fungal strains used in this study $F$. graminearum (52 L, 76 L, 144 L, 149 L), F. culmorum (48 L, 49 L, 70 L, 90 L, 93 L), F. cerealis (33 L, 41 L, 87 L) were all confirmed producers of toxins (3-acetyldeoxynivalenol, 15-acetyl-deoxynivalenol and zearalenone). The chemotype and species determination as well as procedures used for their cultivation, identification and storage were described previously by Dawidziuk et al. (2014). All investigated fungal isolates originated from culture collections of the Functional Evolution of Biological Systems Team (Institute of Plant Genetics, Polish Academy of Sciences, Poznan, Poland).

Bioassays with fungicides

The surface area of the fungal colony was calculated by approximating the mycelium's area to an ellipse by measuring both the length and width of the mycelium. Fungal response to the fungicides was analysed on PDA (growth rate) and Czapek-Dox media (gene expression) amended with flusilazole and carbendazim (all fungicides were added to the liquid medium prior to the casting on the Petri dishes) in simulated day (16 h)/night $(8 \mathrm{~h})$ conditions at $25{ }^{\circ} \mathrm{C}$. The concentration of flusilazole was set to $3 \mathrm{mg} / \mathrm{L}^{-1}$ which was the lowest dose inhibiting fungal growth (Supplementary Fig. 1). The lower concentrations of flusilazole $\left(1 \mathrm{mg} / \mathrm{L}^{-1}\right.$ and $\left.2 \mathrm{mg} / \mathrm{L}^{-1}\right)$ did not significantly influence the growth of the isolates and the higher doses $\left(5 \mathrm{mg} / \mathrm{L}^{-1}\right)$ inhibited growth of all fungal cultures. Carbendazim, belonging to benzimidazoles, was used as a control, to study the response of Fusarium strains to the chemical treatment effectively controlling their growth (no resistant strains was observed and at the same fungicide concentration as in the case of flusilazole treatment $\left(3 \mathrm{mg} / \mathrm{L}^{-1}\right)$, growth of all isolates was inhibited - Supplementary Fig. 2). Each assay was performed in three biological and ten technical replicates. Additional tests were conducted with use of other fungicides with the same mode of action group as flusilazole (metconazole and tebuconazole). Each assay was performed in three biological and ten technical replicates. The concentration of fungicide was set similar to the flusilazole assay. All tested fungicides were analytical grade without any additives and were supplied by the manufacturers in defined concentrations.

Statistical analysis

After testing normal distribution with the Shapiro-Wilk test, the differences in surface area were subjected to analysis of variance (ANOVA), and post hoc means comparisons (Tukey-Kramer honestly significant difference (HSD); $\alpha=0.05$ ). The pairwise differences in gene expression were analysed with Wilcoxon Signed-Ranks non-parametric test $(p \leq 0.05)$. All tests were performed with the Statistica 9.0 software package (Stat Soft, USA).

\section{Isolation of DNA}

Mycelium used for DNA extraction was obtained by inoculating Czapek-Dox broth (Sigma Aldrich, St. Louis, Missouri, USA) with Yeast Extract (Oxoid, Waltham, Massachusetts, USA) and after incubation at $25{ }^{\circ} \mathrm{C}$ on a rotary shaker $(120 \mathrm{rpm})$. Mycelium was collected on filter paper in a Büchner funnel, washed with sterile water, frozen at $-20{ }^{\circ} \mathrm{C}$, and freeze - dried. Total DNA was extracted using the DNeasy Plant Mini Kit (Qiagen, Hilden, Germany). The quality of DNA was estimated by a NanoDrop 2000 UV-vis Spectrophotometer (Thermo Scientific, Wilmington, USA) and a Experion Automated Electrophoresis System (Bio-Rad, Hercules, CA).

Annotation of DHA1 family resistance factors and primer design

For in silico selection of putative DHA1 resistance factors, we used a previously compiled internal database containing available nucleotide and amino acid sequences from model fungi (Popiel et al. 2014) and analysed the complement of MFS transporters encoded by model Fusarium graminearum sequences to find homologs of known DHA1 family resistance factors.

To this end, we selected and clustered MFS transporters from over 140 model fungal genomes, based on 
the MFS_1 domain signature from the Pfam v27 database (Finn et al. 2014); this set was extended by available MFS sequences from TCDB - (Saier et al. 2006) in an approach analogous to Popiel et al. (2014). The unsupervised analysis was carried out in CLANS (Frickey and Lupas 2004). Neural network clustering with default parameters was used to delineate and extract the individual groups of transporters, based on BLASTP E-values below the $1 \mathrm{E}^{-05}$ threshold. Subsequently, the cluster containing 245 MDR/DHA1 sequences, including the known resistance factors (Flr1, caf5, MDR 1 - see Supplementary Fig. 3) was chosen for further analysis. The alignment of 246 sequences (with TPO1, the polyamine transporter from $S$. cerevisiae, as an added out group) was curated with trimal based on a $70 \%$ threshold for column occupancy and 523 positions were retained for phylogeny analysis. The optimal model (LG matrix, 4 rate categories, invariant sites and frequencies estimated on basis of alignment) was selected based on the Bayesian Information Criterion. Both model selection and the subsequent phylogeny reconstruction were done with IQTREE v1.1.4 (support based on ultra-fast bootstrap estimation, stopping at 216 trees due to convergence (Nguyen et al. 2014)). On the basis of the reconstructed phylogeny two candidate DHA1 transporters were selected - one present in Fusarium graminearum/pseudograminearum (FGSG_02865), the second present in a number of Hypocreales genomes including the other phytopathogenic fusaria (FGSG_05318).

For the two homologs, sets of degenerate, crossspecies specific primers were designed by targeting their conserved protein sequence features. The primers were screened against propensity for homodimer and heterodimer formation on the basis of nearest neighbour energy/melting temperature assessment. The design and testing were carried out with both IDT OligoAnalyzer (http://eu.idtdna.com/calc/analyzer) and in-house Python scripts implementing the nearestneighbour enthalpy/entropy approximations (Owczarzy et al. 2008).

PCR amplification, sequencing, RNA isolation and gene expression data analysis

The protocols for PCR and sequencing conditions have been previously described by Popiel et al. (2014). Fungal isolates were incubated for 4 days before addition of fungicides $\left(3 \mathrm{mg} / \mathrm{L}^{-1}-\right.$ the same fungicide concentration as in the growth rate experiment, necessary for comparison of the individual parts of the experiment). Every $24 \mathrm{~h}$ after chemical treatment the mycelium was collected from the Czapek-Dox medium and each sample was weighed on a laboratory scale (Sartorius AG, Göttingen, Germany). Total RNA from chosen, representative isolates was purified using an RNeasy Plant Mini Kit (Qiagen, Hilden, Germany) according to the manufacturers' protocol with the additional DNase digestion step. The quality of total RNA was estimated by Nanodrop (Thermo Scientific, Wilmington, DE) and via Bioanalyzer (Bio-Rad, Hercules, CA). RNA dissolved in DEPC water was stored at $-80{ }^{\circ} \mathrm{C}$. RT-PCR primers were designed on the basis of previously sequenced gene fragments using Primer 3 (Rozen and Skaletsky 2000) and their properties were tested using OligoCalc (Supplementary Table 1) (Kibbe 2007).

Real time RT-PCR was used to amplify FGSG_02865, Tri5 (trichothecene synthase), Tri6 (trichothecene transcription factor), Tri12 (trichothecene efflux pump), Zea2 (zearalenone synthase), Zeb2 (zearalenone transcription factor), Zral (zearalenone efflux pump) (Desjardins and Proctor 2007; Lee et al. 2011) genes and the constitutive Tub2 ( $\beta$-tubulin) and Act (actin) genes from each RNA sample of the fungal strains.

Real time RT-PCR reactions were performed using an CFX96 Touch $^{\text {TM }}$ Real-Time PCR Detection System (Bio-Rad, Hercules, CA). Analyses were conducted using iTaq One Step SYBR Green RT-qPCR Kit (BioRad, Hercules, California, USA). The total reaction volume was $25 \mu \mathrm{l}$ : $12.5 \mu \mathrm{l}$ iTaq One Step SYBR Green RT-qPCR mix, $1 \mu$ RNA ( $<35$ ng), $0.5 \mu l$ each primer $(10 \mu \mathrm{M}), 0.125 \mu \mathrm{l}$ reverse transcriptase and $5.125 \mu \mathrm{l}$ nuclease free water. The reaction was carried out using the following protocol: initial denaturation $94{ }^{\circ} \mathrm{C}$ for $2 \mathrm{~min}$, followed by 40 cycles at $94{ }^{\circ} \mathrm{C}$ for $15 \mathrm{~s}, 59^{\circ} \mathrm{C}$ for $1 \mathrm{~min}$. In the experiment, we used three biological and two technical replicates together with a template-free negative control in each analysis of both target and control genes. The melting curve analysis (from 70 to $95{ }^{\circ} \mathrm{C}$ ) confirmed primer pair specificity. As a control, we used mycelium samples cultivated on medium without the addition of fungicides. Relative quantification of gene expression was calculated using the $2^{-\Delta \Delta \mathrm{Ct}}$ method (Bio-Rad, Hercules, CA). Data from samples treated with flusilazole and carbendazim were normalized to actin and $\beta$-tubulin genes as internal 
controls (Real-Time PCR Application Guide, Bio-Rad, Hercules CA).

\section{Determination of trichothecenes and zearalenone concentration}

Fungal isolates were incubated for 4 days before addition of fungicides $\left(3 \mathrm{mg} / \mathrm{L}^{-1}\right.$ - the same fungicide concentration as in the growth rate experiment necessary for comparison of the individual parts of the experiment). Every $24 \mathrm{~h}$ after chemical treatment the mycelium was collected from the Czapek-Dox medium and each sample was weighed on a laboratory scale (Sartorius AG, Göttingen, Germany). Determination of trichothecenes was performed in solid PDA culture. Briefly, subsamples ( $1 \mathrm{~g}$ of mycelium with medium) were extracted with acetonitrile/water (82:18) and cleaned-up on a Myco Sep 227 Trich + and ZearalaTest affinity columns. The analyses of trichothecenes were run on a gas chromatograph (Hewlett Packard GC 6890) hyphenated to a mass spectrometer (Hewlett Packard 5972 A, Waldbronn, Germany), using an HP-5MS, $0.25 \mathrm{~mm} \times$ $30 \mathrm{~m}$ capillary column. Quantitative analysis was performed in single ion monitored mode and qualitative analysis was performed in SCAN mode (100-700 amu). Recoveries for DON was $84 \pm 3.8 \%$. Limit of detection was $0.01 \mathrm{mg} \mathrm{kg}^{-1}$.

The HPLC analyses of trichothecenes were run on Waters HPLC 2695 apparatus with a Waters 2475 Multi $\lambda$ Fluorescence Detector and a Waters 2996 Array
Detector. Separation was achieved on a $150 \mathrm{~mm}$ length $\times 3.9 \mathrm{~mm}$ diameter Nova Pak C-18, $4 \mu \mathrm{m}$ particle size column and eluted with acetonitrile- water-methanol $(46: 46: 8, \mathrm{v} / \mathrm{v} / \mathrm{v})$ at a flow rate of $0.5 \mathrm{ml} / \mathrm{min}$. ZEA was detected with a Waters 2475 Multi $\lambda$ Fluorescence Detector and the excitation and emission wavelengths were 274 and $440 \mathrm{~nm}$, respectively. Estimation of ZEA was performed by a comparison of peak areas with those of an external standard ( $>95 \%$, Aldrich, Milwaukee, USA) or by co-injection with a standard. The detection limit of ZEA was $3 \mathrm{ng} \mathrm{g}^{-1}$.

\section{Results}

Screening for resistant fungal strains

Fungicide sensitivity assays were performed on a set of diverse fungal strains (Fusarium graminearum, F. culmorum, F. cerealis) that have shown a significant decrease of growth rate of most isolates treated with flusilazole and almost total growth suppression by carbendazim (Supplementary Fig. 2). All tested F. culmorum (48 L, 49 L, 70 L, 90 L, 93 L) and F. cerealis $(33 \mathrm{~L}, 41 \mathrm{~L}, 87 \mathrm{~L})$ isolates were susceptible to flusilazole in the concentration of $3 \mathrm{mg} / \mathrm{L}^{-1}$ and their surface area was reduced by at least sixty percent (Fig. 1). The response toward fungicide differ among F. graminearum strains and while the $76 \mathrm{~L}$ and $149 \mathrm{~L}$ isolate samples were moderately affected (mean decrease of $27 \%$ in surface area relative to control

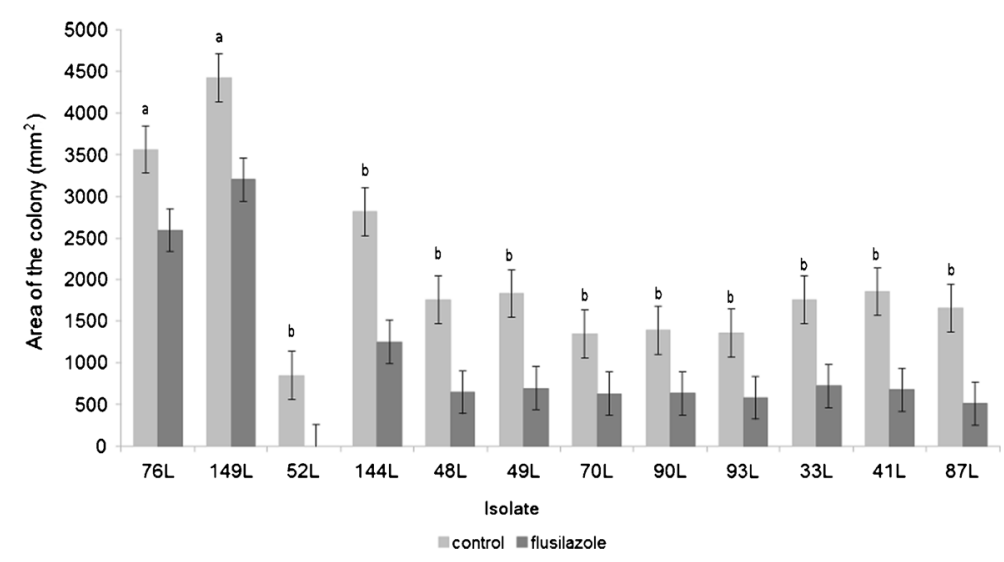

Fig. 1 Surface area (5th day) of the Fusarium isolates non-treated and treated with flusilazole $\left(3 \mathrm{mg} / \mathrm{L}^{-1}\right): 52 \mathrm{~L}, 76 \mathrm{~L}, 144 \mathrm{~L}, 149 \mathrm{~L}-$ F. graminearum, 48 L, 49 L, 70 L, 90 L, 93 L - F. culmorum, 33 L, $41 \mathrm{~L}, 87 \mathrm{~L}-F$. cerealis. Bars indicate standard deviation of the

mean. $\mathrm{a}$ is used to point out the isolates showing no significant differences at $\alpha=0.05$; $b$ is used to point out the isolates showing significant differences at $\alpha=0.05$ 
samples; Fig. 1), the growth of strain $52 \mathrm{~L}$ was completely inhibited. While bulk of testing was conducted with two fungicides (flusilazole and carbendazim), additional tests were conducted with other fungicides (tebuconazole and metconazole). Performed additional experiments have shown that metconazole and tebuconazole treatment was slightly more effective in the bioassays in the tested isolates than flusilazole. Tested isolates were most susceptible to the tebuconazole but observed growth changes were not statistically significant. Tested isolates did not express increased resistance towards those two fungicides regardless of its concentration (Supplementary Figs. 5 and 6).

\section{Phylogenetic and bioinformatic characterisation of two candidate DHA1 transporters}

Based on the rooted phylogeny of the DHA1 cluster (presence of strongly supported Basidiomycete sister clades at varying depths of the tree), the two Fusarium graminearum DHA1 genes (FGSG_02865, FGSG_05318) likely diverged before the split between Basidiomycetes and Ascomycetes (Supplementary Fig. 3). Neither gene has clearly orthologous relationships to the known resistance factors of yeasts (caf5, FLR1, CYHR, MDR1), which form a strongly supported

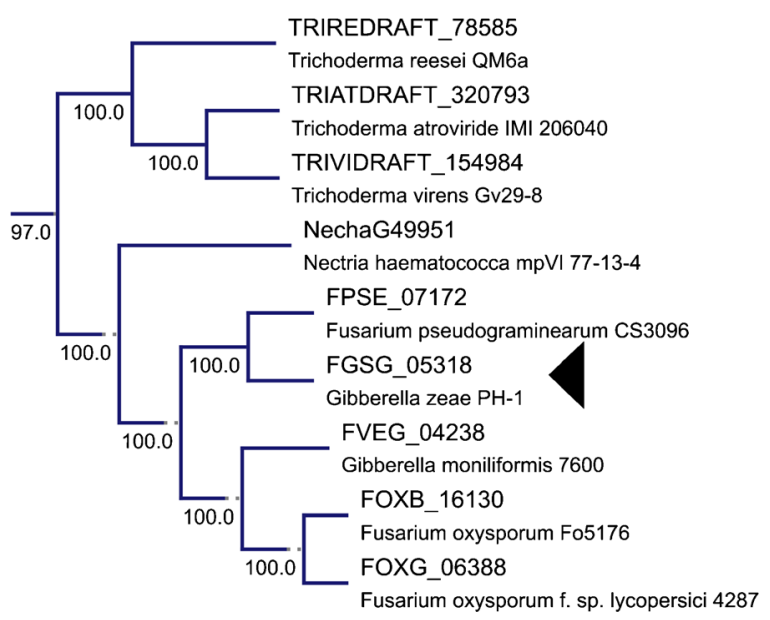

Fig. 2 Maximum-likelihood consensus phylogeny of FGSG_05318 (Fusarium graminearum multidrug transporter belonging to DHA1 family). The monophyletic clades were extracted from the full MDR/DHA1 phylogenetic tree (consensus tree, clades with support of $<70 \%$ collapsed) with ETE2 (see Supplementary Fig. 3 for the original tree of 246 sequences) monophyletic clade exclusive to Saccharomycetes and Schizosaccharomycetes. While FGSG_05318 is present in a number of Hypocreales species (Fig. 2), the FGSG_02865 gene lacks orthologs in other Hypocreales and appears to be closely related to transporters retained mostly in Eurotiomycete and Dothideomycete species (including Mycosphaerella graminicola and Pyrenophora tritici - known cereal pathogens - see Fig. 3).

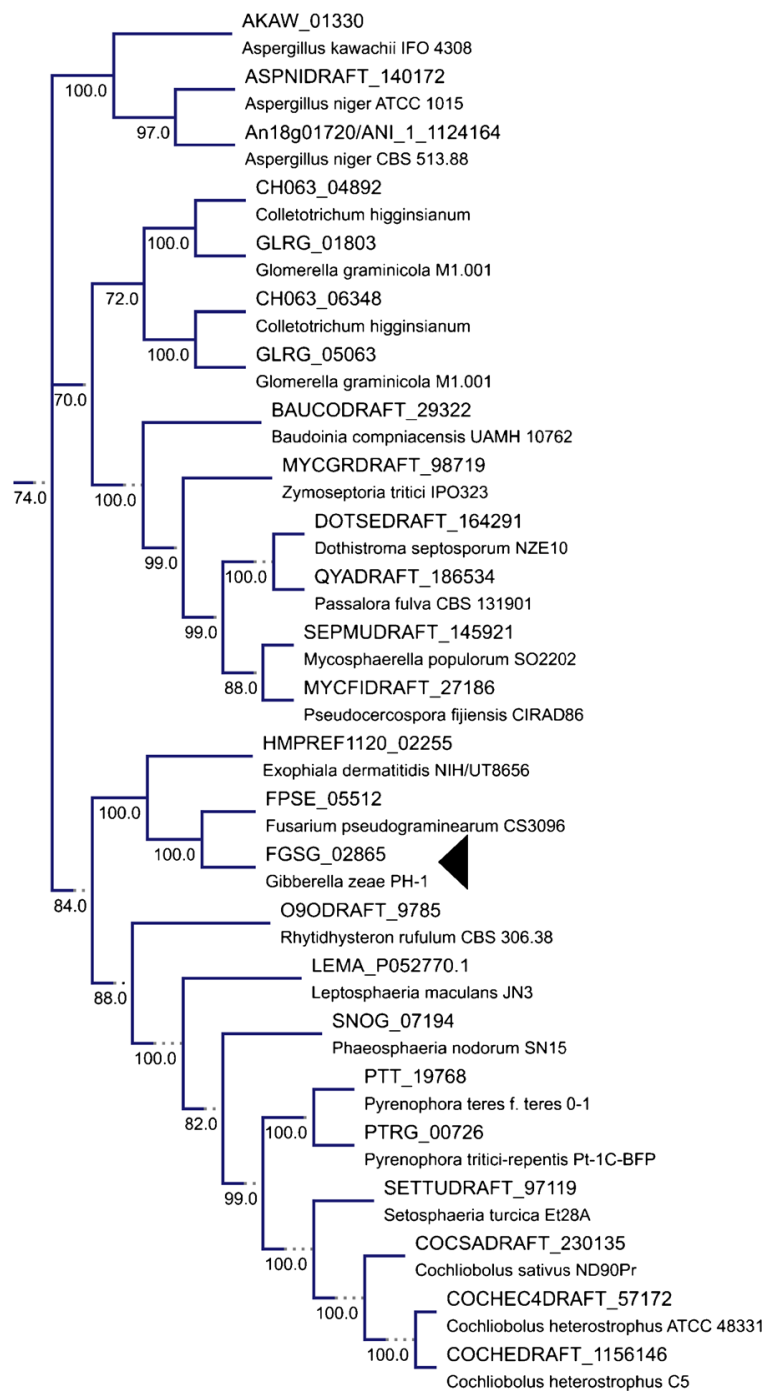

Fig. 3 Maximum-likelihood consensus phylogeny of candidate gene FGSG_02865 (Fusarium graminearum multidrug transporter belonging to DHA1 family). The monophyletic clades were extracted from the full MDR/DHA1 phylogenetic tree (consensus tree, clades with support of $<70 \%$ collapsed) with ETE2 (see Supplementary Fig. 3 for the original tree of 246 sequences) 
Sequencing of DHA1 family members

The cross-species specific primers allowed us to amplify parts of the FGSG_02865 gene and its orthologs in Fusarium graminearum (52 L - GenBank Accession KP721339.1, 76 L - KP721340.1, 149 L - KP721341.1 ), F. culmorum (93 L - KP721337.1), F. cerealis (33 L KP721336.1). The obtained amplicons are part of the second exon of the gene. The differences between sequences in the Fusarium strains have shown no significant changes in the nucleotide or protein sequences (i.e. nonsynonymous substitutions possibly leading to changes in hydrophobicity profile and resulting disruption of transmembrane segments).

DHA1 transporter expression in fungicide-treated cultures

The expression of both FGSG_02865 and FGSG_05318 was tested via quantitative RT-PCR (with $\beta$-tubulin and actin as reference genes). In keeping with observed culture growth, the more resistant isolates from $F$. graminearum ( $76 \mathrm{~L}$ and $149 \mathrm{~L}$ ) exhibited almost 2 -fold $(P \leq 0.05)$ induced upregulation of the first gene (Fig. 4) while the level of the transcript in $52 \mathrm{~L}$ isolate (very susceptible to flusilazole treatment) was decreased (0.4-fold expression at 3rd day after exposition). The FGSG_02865 was also upregulated in the presence of carbendazim in all tested isolates except $52 \mathrm{~L}$. This upregulation was first observed $24 \mathrm{~h}$ after treatment and the transcript levels remained elevated. The FGSG_05318 transcript levels remained constant across all conditions. In the cultures treated with metconazole and tebuconazole, expression of FGSG_02865 gene was slightly increased, but the results were not statistically significant (Supplementary Fig. 7).

\section{Expression of biosynthetic genes and mycotoxin concentration in fungicide-treated cultures}

The RT-PCR expression profiling of key mycotoxin biosynthetic genes (trichothecene - Fig. 5 and zearalenone - Fig. 6 biosynthesis genes in F. graminearum $(\mathrm{Fg}$ - mean of $149 \mathrm{~L}, 76 \mathrm{~L}$ and $52 \mathrm{~L}$ isolates) and F. culmorum ( $\mathrm{Fc}-93 \mathrm{~L}$ ) has shown changes in expression (upregulation) in the isolates of two most frequent pathogens ( $F$. graminearum and F. culmorum) (detailed informations in Supplementary Fig. 4).
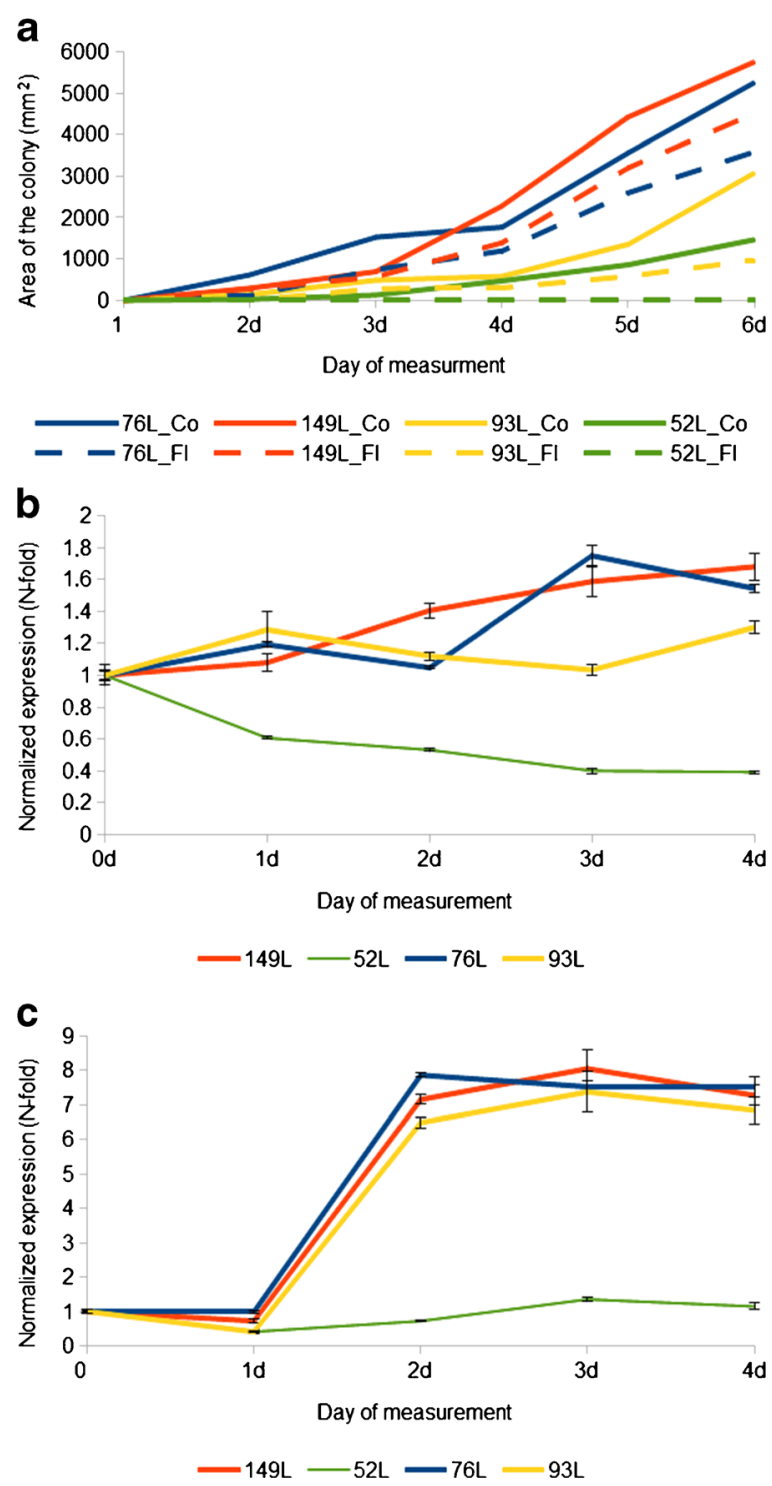

Fig. 4 Response of Fusarium isolates to fungicide treatment: a surface area of the mycelium treated with flusilazole. Isolates $52 \mathrm{~L}$, $76 \mathrm{~L}, 149 \mathrm{~L}-F$. graminearum, $93 \mathrm{~L}-F$. culmorum. Treatments: $\mathrm{Co}$ control without fungicide, $\mathrm{Fl}$ flusilazole (treatment with carbendazim not shown due to complete growth inhibition), b expression of FGSG_02865 gene after flusilazole treatment (control samples not treated with fungicide not shown due to the lack of significant changes in gene expression), c expression of FGSG_02865 gene after carbendazim treatment (control samples not treated with fungicide not shown due to the lack of significant changes in gene expression). Each experiment performed in two biological and three technical replicates. Bars indicate standard deviation of the mean

In the presence of carbendazim, both the core gene (Tri5/Zea2) and the key transcription factor (Tri6/Zeb2) 

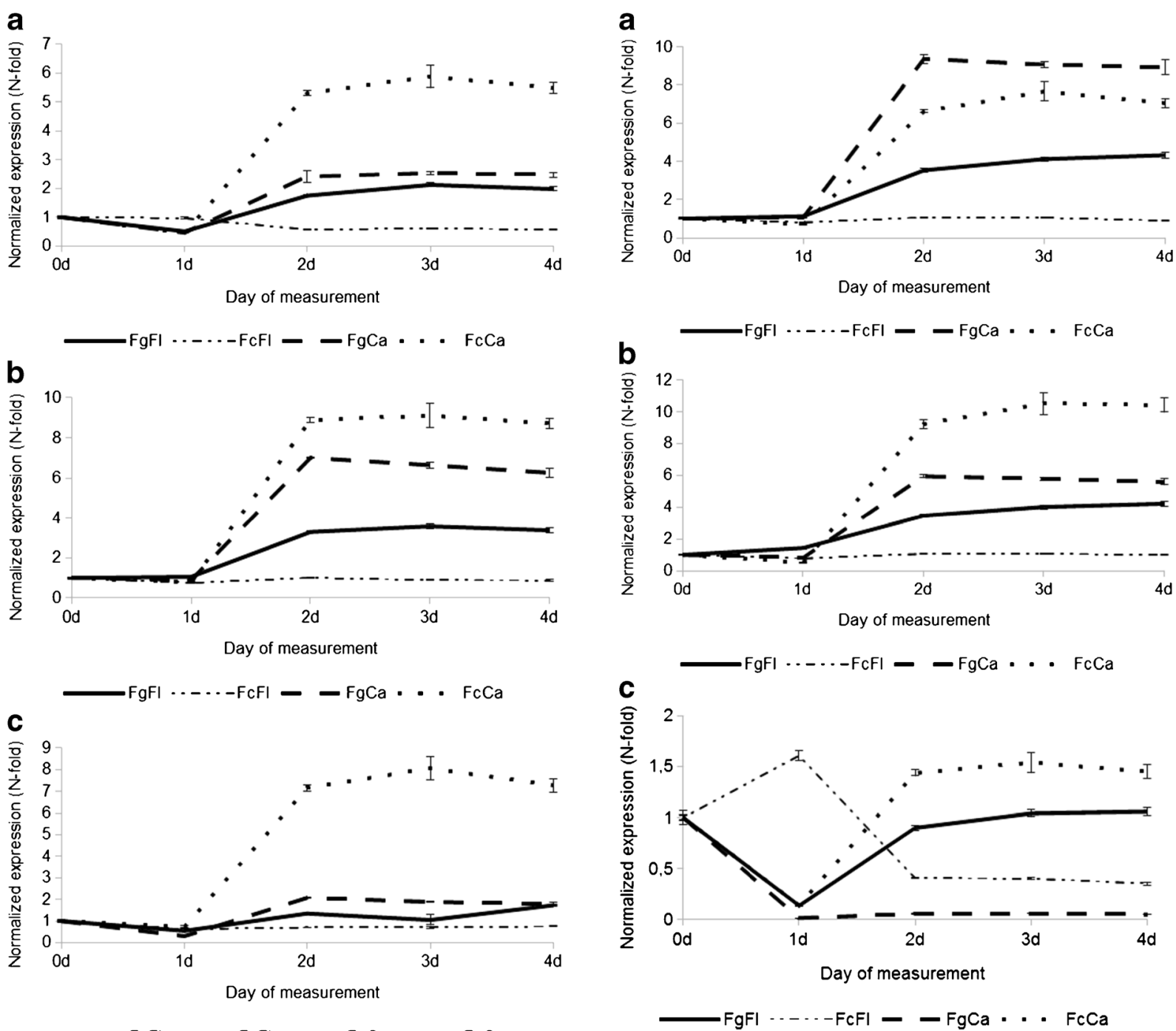

Fig. 5 Expression of trichothecene biosynthesis genes in F. graminearum (Fg - mean of $149 \mathrm{~L}, 76 \mathrm{~L}$ and $52 \mathrm{~L})$ and $F$. culmorum $(\mathrm{Fc}-93 \mathrm{~L})$ isolates after flusilazole $(F l)$ and carbendazim ( $\mathrm{Ca}$ ) treatment. a Tri5 gene expression, b Tri6 gene expression, c Tri12 gene expression. Control samples not treated with fungicide not shown due to the lack of significant changes in gene expression. Each experiment performed in two biological and three technical replicates. Bars indicate standard deviation of the mean

Fig. 6 Expression of zearalenone biosynthesis genes in F. graminearum ( $\mathrm{Fg}$ - mean of $149 \mathrm{~L}, 76 \mathrm{~L}$ and $52 \mathrm{~L}$ ) and F. culmorum $(\mathrm{Fc}-93 \mathrm{~L})$ isolates after flusilazole $(F l)$ and carbendazim $(\mathrm{Ca})$ treatment. a Zea2 gene expression, b Zeb2 gene expression, c Zral gene expression. Control samples not treated with fungicide not shown due to the lack of significant changes in gene expression. Each experiment performed in two biological and three technical replicates. Bars indicate standard deviation of the mean

have shown significantly $(P \leq 0.05)$ increased transcriptional activity. Strongest induction was observed in carbendazim treatment of $F$. culmorum isolate, in which the relative normalized expression of biosynthetic genes reached very high levels (Tri5 5.30 fold, Tri $6-9.11$, Tri12 - 8.05, Zea2 - 7.68,

Zeb2 - 10.52, Zral - 1.53). The F. graminearum isolates have likewise exhibited increased expression of all tested genes.

The treatment with a less potent fungicide flusilazole caused significantly $(P \leq 0.05)$ increased expression of both trichothecene and zearalenone biosynthetic genes in F. graminearum (Tri5 - 2.12 fold, Tri 6- 
3.58 fold, Tri12 - 1.04 fold, Zea2 - 4.12 fold, Zeb2 4.00 fold, Zral - 1.04 at 3rd day after exposition) however no such changes were observed in the resistant F. culmorum isolate.

There were no significant changes in expression patterns of biosynthetic genes between resistant and susceptible $F$. graminearum strains (Supplementary Fig. 4). Interestingly, the resistant $F$. graminearum strain has shown visible, morphological changes in the surviving mycelium, indicating secondary metabolite biosynthesis in response to carbendazim treatment. Liquid cultures treated with carbendazim after $24 \mathrm{~h}$ began to change color suggesting the accumulation of pigments in the culture; there was while no visible reaction in response to flusilazole (Fig. 7).

Chemical analyses have shown that in concentration of DON and ZEN in the samples treated with flusilazole was higher than in the control samples e.g. in $52 \mathrm{~L}$ isolate concentration of DON and ZEN in the control sample was respectively 60.02 and $19.99 \mathrm{ng} / \mu \mathrm{l}$ and after 3 days after fungicide exposition: 82.48 and $18.65 \mathrm{ng} /$ $\mu 1$. Results indicate that remaining viable fungal cells after chemical treatment are still able to produce mycotoxins. Interestingly similar results were obtained after treatment with carbendazim (e.g. $52 \mathrm{~L}$ isolate; control DON $60.02 \mathrm{ng} / \mathrm{ml}$, ZEN $19.99 \mathrm{ng} / \mathrm{ml}$; carbendazim treatment after 3 days DON $71.85 \mathrm{ng} / \mathrm{ml}$ and ZEN $25.56 \mathrm{ng} / \mathrm{ml}$ ) which effectively reduced growth rate of all tested isolates. Details are given in Supplementary Table 2.

\section{Discussion}

Multidrug resistance (MDR) can be characterized as the acquisition of the simultaneous partial or complete resistance to the toxic compounds differing both chemically and structurally (Sá-Correia and Tenreiro 2002). This in turn contributes to the problems associated with effectively controlling the fungal phytopathogens, where improved resistance to fungicidal compounds is becoming more frequent (Ma and Michailides 2005; Chen and Zhou 2009).

In our study, several $F$. graminearum and F. culmorum isolates have shown increased resistance to the triazole fungicide flusilazole. Previous studies have shown the involvement of transporter genes in development of azole resistance, as supported by transcriptomic studies and deletion mutants. The pertinent examples ( $\mathrm{ABC}$ transporter superfamily) include among others: A. fumigatus Mdrl and A. nidulans AtrD (Andrade et al. 2000), Botrytis cinerea BcatrB (Vermeulen et al. 2001). The major facilitator superfamily (MFS) transporter involvement was also noted in other plant pathogenic species e.g. in Botrytis cinerea (Bcmfs1 - Hayashi et al. 2002) and Zymoseptoria tritici (MgMFS1 - Omrane et al. 2015).

In case of fusaria, the recent experiments performed by (Abou Ammar et al. 2013) on the tebuconazole
Fig. 7 Production of pigment by full-grown $F$. graminearum $(76 \mathrm{~L})$ culture treated with carbendazim and flusilazole $\left(3 \mathrm{mg} / \mathrm{L}^{-1}\right)$ : a strain without carbendazim, b strain without flusilazole, c strain $24 \mathrm{~h}$ after carbendazim treatment, $\mathbf{d}$ strain $24 \mathrm{~h}$ after flusilazole treatment
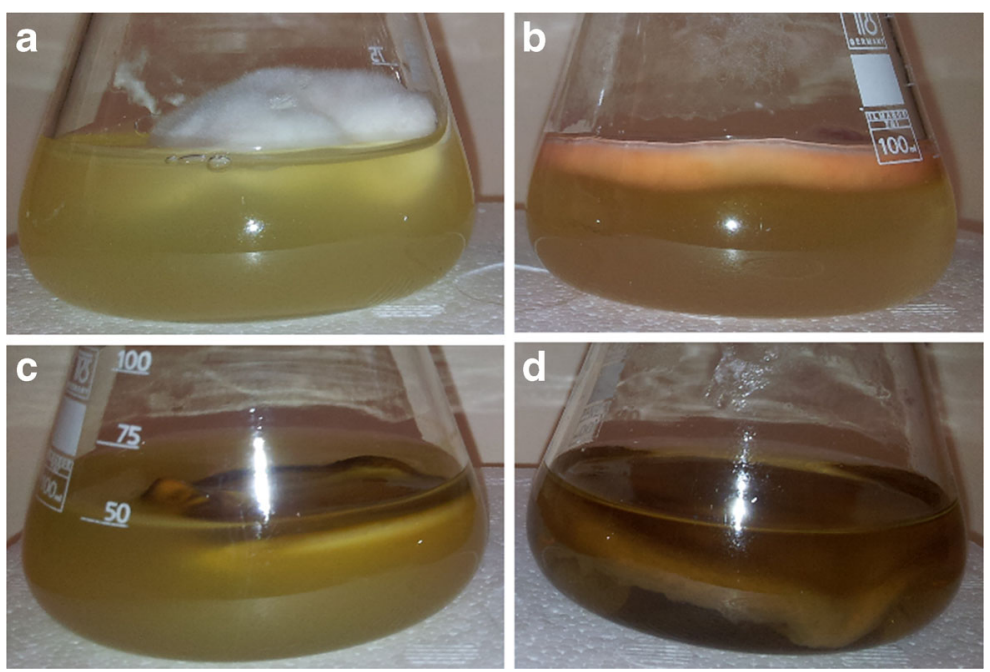
resistant strains of $F$. graminearum highlighted the particular involvement of $\mathrm{ABC}$ transporters: $\mathrm{FgABC} 3$ (FGSG_04580; PDR) and FgABC4 (FGSG_17058; MRP). With regards to possible MFS/DHA1 resistance factors investigated here, both phylogenetic and expression parts of our analysis point to divergent functionality of their relatives in fusaria,as compared to yeasts. The distant homologs of yeast resistance factors do not appear to be strongly upregulated in early response to fungicide treatments.

However, the results support the possible involvement of FGSG_02865 gene as part of the general response to fungicide treatment (possibly in reaction to the resulting oxidative stress). This is supported by the finding that higher expression was consistently observed in the cultures subjected to carbendazim treatment; where the strong transcriptional response is not associated with observed resistance toward the fungicide, but is combined with upregulation of mycotoxin biosynthetic genes. Notably, in the context of our study, carbendazim has proven to be a very effective fungicide (Supplementary Fig. 2). However, there are past reports of resistant strains found in the field (Chen et al. 2008; Chen and Zhou 2009) or artificially created via sitedirected mutagenesis of codon 167 in beta-tubulin (Zhang et al. 2009b).

The activity of multiple, broad-specificity transporters, indicates that those proteins may complement each other (Popiel et al. 2014; Del Sorbo et al. 2000) particularly when gene family members are adapted differently in divergent species, as our analysis suggests. In case of more effective fungicides, this is likely coupled to involvement of multiple transporters also in the reaction to the inevitable stress associated with cell breakdown and release of both reactive oxygen species and multiple chemicals.

Importantly, the efflux mechanisms can also synergise with other, stronger factors. In the case of azole resistance, the point mutations in $14 \alpha$-sterol demethylase seem to be such a crucial determinant (Fan et al. 2013), while transporter activity contributes an additional quantitative reaction. As such, disabling, or partially suppressing, one of the several putative efflux pumps would only decrease the resistance of fungal strains and in most cases would not cause full susceptibility. An analogous example is provided by natural trichothecene toxins. There, the activity of trichothecene 3-O-acetyltransferase encoded by the gene Tri101 seems to be the main protection factor against trichothecenes in non-producing Fusarium species (Kimura et al. 2003), whereas transporter encoded by the gene Tri12 acts complementary to the main mechanism and is retained mostly in producer species.

The multifaceted reaction of fungi to fungicide treatments is not limited to the coping mechanisms of efflux or resistance-associated mutations of targeted genes. The stress of fungicidal treatments affects other facets of fungal metabolism, including sporulation (e.g. Miguel et al. 2015) and secondary metabolite accumulation (e.g. Ochiai et al. 2007; Mateo et al. 2011; Marín et al. 2013). The possible influence on the activity of the biosynthetic genes responsible for the production of mycotoxins can thus affect the efficiency of fungicidal treatments in controlling mycotoxin producers. The present evidence suggests this influence can differ considerably between phytopathogenic strains of different descent.

In the tested samples, the presence of flusilazole did not affect expression of key trichothecene and zearalenone biosynthetic genes in F. culmorum, while F. graminearum isolates responded by enhanced activity of Tri5, Tri6, Tri12, Zea2, Zeb2 and Zra1. Interestingly, both species react stronger in the presence of the much more effective benzimidazole fungicide (carbendazim), where the observed morphological changes in F. graminearum support a generalised reaction to a strong stressor.

Importantly, our results are consistent with earlier works of Ochiai et al. (2007), stating that non-lethal doses of triazole fungicides activate the Tri5 gene (trichothecene synthase) in Fusarium graminearum. Similar research performed on Fusarium verticillioides and Fusarium proliferatum strains has shown that sublethal doses of fungicides in combination with mild water stress and temperatures less than $35^{\circ} \mathrm{C}$, significantly induced Fum1 expression with slight differences in both species (Marín et al. 2013). Analogous studies carried out with different fungicides (tebuconazole and prochloraz) on $F$. langsethiae revealed an analogous increase of toxin production at low doses of some fungicides with regard to untreated cultures (Mateo et al. 2011).

However, the additional observation in comparison with the above works is that even a very effective treatment (complete growth suppression by carbendazim) can still result in the induction of mycotoxin biosynthetic genes, and possibly increase accumulation of mycotoxins. 
Indeed, our in vitro analysis has shown that addition of carbendazim $\left(3 \mathrm{mg} / \mathrm{L}^{-1}\right)$ to the medium inoculated with Fusarium species caused complete growth inhibition of all tested isolated. Still, the same fungicide ( $3 \mathrm{mg} / \mathrm{L}^{-1}$ ) added to medium with mycelium in the stationary growth stage caused enhanced production of toxins by the surviving mycelium. These inferences are supported by morphological changes in mycelium ( $F$. graminearum), indicating very strong reaction to benzimidazoles (pigment accumulation, not observed after flusilazole treatment). The visible reaction is conjoined with activation of the transporter(s) potentially involved in the efflux of harmful substances as part of the generalised reaction to stressor stimulus (FGSG_02865), as well as increased activity of toxin biosynthetic genes. While previous inquiries in the context of fusarial benzimidazole resistance have suggested that resistant strains increase toxin accumulation posttreatment (Zhang et al. 2009a), our results suggest that sensitive strains could affect the change as well, as long as enough mycelium is present.

In summary, it seems that effectiveness of fungicidal treatments should be considered depending not only on the amount and type of antifungal substance (where enhanced production of toxins is caused mainly by low doses/less effective fungicides), but also on the timing of chemical treatment relative to the growth phase. The amount of already present mycelium, where a strong fungicide leaves a number of viable or semiviable cells, could translate to higher levels of contamination. This conjecture is supported by some of the previously published studies on fusarial head blight, where the differences in effectiveness of fungicides and/or treatment timings on mycotoxin content were apparent (e.g. tebuconazole and prochloraz - Matthies and Buchenauer 2000, thiophanate - Yoshida et al. 2012). However, while results of these inquiries have shown the benefits of optimised timing, the discrepancies between different studies are only rarely investigated in conjunction with molecular studies such as expression profiling of mycotoxin biosynthetic genes under different regimes (e.g. Marín et al. 2013) or prediction of regulatory single nucleotide polymorphisms affecting treatment efficiency (e.g. Talas et al. 2016).

In view of the above, the early detection of disease incidence and characterisation of secondary risks (mycotoxin accumulation post-treatment, increased fungicide tolerance as opposed to complete resistance) is reinforced as a critical component of successful plant protection practices. Further elucidation of causal links (transcriptional and metabolic responses to treatment, regulatory mechanisms) in different phytopathogenic fungi will shape the practical impact of such research on selection and deployment of fungicide treatments in the field.

Acknowledgments This work was supported by: "Molecular diagnostics of fungicide resistance in phytopathogenic fungi", The National Centre for Research and Development, LIDER/27/ 204/L-3/11/NCBR/2012" research grant.

\section{Compliance with ethical standards}

Conflict of interest The authors declare that they have no conflict of interest.

Open Access This article is distributed under the terms of the Creative Commons Attribution 4.0 International License (http:// creativecommons.org/licenses/by/4.0/), which permits unrestricted use, distribution, and reproduction in any medium, provided you give appropriate credit to the original author(s) and the source, provide a link to the Creative Commons license, and indicate if changes were made.

\section{References}

Alarco, A. M., Balan, I., Talibi, D., Mainville, N., \& Raymond, M. (1997). AP1-mediated multidrug resistance in Saccharomyces cerevisiae requires FLR1 encoding a transporter of the major facilitator superfamily. Journal of Biological Chemistry, 272, 19304-19313.

Ammar, G. A., Tryono, R., Döll, K., Karlovsky, P., Deising, H. B., \& Wirsel, S. G. (2013). Identification of ABC transporter genes of Fusarium graminearum with roles in azole tolerance and/or virulence. PLOS ONE, 8, e79042.

Andrade, A. C., Del Sorbo, G., Van Nistelrooy, J. G., \& De Waard, M. A. (2000). The ABC transporter AtrB from Aspergillus nidulans mediates resistance to all major classes of fungicides and some natural toxic compounds. Microbiology, 146, 1987-1997.

Becher, R., Weihmann, F., Deising, H. B., \& Wirsel, S. G. (2011). Development of a novel multiplex DNA microarray for Fusarium graminearum and analysis of azole fungicide responses. BMC Genomics, 12, 52.

Benko, Z., Fenyvesvolgyi, C., Pesti, M., \& Sipiczki, M. (2004). The transcription factor Pap1/Caf3 plays a central role in the determination of caffeine resistance in Schizosaccharomyces pombe. Molecular Genetics and Genomics, 271, 161-170.

Chen, Y., \& Zhou, M. G. (2009). Characterization of Fusarium graminearum isolates resistant to both carbendazim and a new fungicide JS399-19. Phytopathology, 99, 441-446.

Chen, C. J., Bi, C. W., Yu, J. J., Wang, J. X., Li, H. X., Luo, Q. Q., \& Zhou, M. G. (2008). Carbendazim-resistance and its 
molecular mechanism in Gibberella zeae. Journal of Plant Pathology, 90, 69.

Cools, H. J., Hawkins, N. J., \& Fraaije, B. A. (2013). Constraints on the evolution of azole resistance in plant pathogenic fungi. Plant Pathology, 62, 36-42.

Costa, C., Dias, P. J., Sá-Correia, I., \& Teixeira, M. C. (2014). MFS multidrug transporters in pathogenic fungi: do they have real clinical impact. Front Physiology, 5, 197.

Dawidziuk, A., Koczyk, G., Popiel, D., Kaczmarek, J., \& Busko, M. (2014). Molecular diagnostics on the toxigenic potential of Fusarium spp. plant pathogens. Journal of Applied Microbiology, 116, 1607-1620.

Deising, H. B., Reimann, S., \& Pascholati, S. F. (2008). Mechanisms and significance of fungicide resistance. Brazilian Journal of Microbiology, 39, 286-295.

Del Sorbo, G., Schoonbeek, H. J., \& De Waard, M. A. (2000). Fungal transporters involved in efflux of natural toxic compounds and fungicides. Fungal Genetics and Biology, 30, 115.

Desjardins, A. E., \& Proctor, R. H. (2007). Molecular biology of Fusarium mycotoxins. International Journal of Food Microbiology, 119, 47-50.

D’mello, D., Mehta, D., Pereira, J., \& Rao, C. V. (1999). A toxicity study of simultaneous administration of Tamoxifen ${ }^{\circledR}$ and Diazepam ${ }^{\circledR}$ to female Wistar rats. Experimental and Toxicologic Pathology, 51, 549-553.

Fan, J., Urban, M., Parker, J. E., Brewer, H. C., Kelly, S. L., Hammond-Kosack, K. E., et al. (2013). Characterization of the sterol $14 \alpha$-demethylases of Fusarium graminearum identifies a novel genus-specific CYP51 function. New Phytologist, 198, 821-835.

Finn, R. D., Bateman, A., Clements, J., Coggill, P., Eberhardt, R. Y., Eddy, S. R., et al. (2014). Pfam: the protein families database. Nucleic Acids Research, 42, D222-D230.

Fling, M. E., Kopf, J., Tamarkin, A., Gorman, J. A., Smith, H. A., \& Koltin, Y. (1991). Analysis of a Candida albicans gene that encodes a novel mechanism for resistance to benomyl and methotrexate. Molecular and General Genetics, 227, 318-329.

Frickey, T., \& Lupas, A. (2004). CLANS: a Java application for visualizing protein families based on pairwise similarity. Bioinformatics, 20, 3702-3704.

Hayashi, K., Schoonbeek, H. J., \& De Waard, M. A. (2002). Bcmfs1, a novel major facilitator superfamily transporter from Botrytis cinerea, provides tolerance towards the natural toxic compounds camptothecin and cercosporin and towards fungicides. Applied and Environmental Microbiology, 68, 4996-5004.

Kibbe, W. A. (2007). OligoCalc: an online oligonucleotide properties calculator. Nucleic Acids Research, 35, W43-W46.

Kimura, M., Tokai, T., Matsumoto, G., Fujimura, M., Hamamoto, H., Yoneyama, K., et al. (2003). Trichothecene nonproducer Gibberella species have both functional and nonfunctional 3O-acetyltransferase genes. Genetics, 163, 677-684.

Lee, S., Son, H., Lee, J., Lee, Y. R., \& Lee, Y. W. (2011). A putative $\mathrm{ABC}$ transporter gene, ZRA1, is required for zearalenone production in Gibberella zeae. Current Genetics, 57, 343-351.

Liu, X., Jiang, J., Shao, J., Yin, Y., \& Ma, Z. (2010). Gene transcription profiling of Fusarium graminearum treated with an azole fungicide tebuconazole. Applied Microbiology and Biotechnology, 85, 1105-1114.

Ma, Z., \& Michailides, T. J. (2005). Advances in understanding molecular mechanisms of fungicide resistance and molecular detection of resistant genotypes in phytopathogenic fungi. Crop Protection, 24, 853-863.

Marín, P., de Ory, A., Cruz, A., Magan, N., \& González-Jaén, M. T. (2013). Potential effects of environmental conditions on the efficiency of the antifungal tebuconazole controlling Fusarium verticillioides and Fusarium proliferatum growth rate and fumonisin biosynthesis. International Journal of Food Microbiology, 165, 251-258.

Mateo, E. M., Valle-Algarra, F. M., Mateo, R., Jimenez, M., \& Magan, N. (2011). Effect of fenpropimorph, prochloraz and tebuconazole on growth and production of T-2 and HT-2 toxins by Fusarium langsethiae in oat-based medium. International Journal of Food Microbiology, 151, 289-298.

Matthies, A., \& Buchenauer, H. (2000). Effect of tebuconazole (Folicur $\left.{ }^{\circledR}\right)$ and prochloraz (Sportale $\left.®\right)$ treatments on Fusarium head scab development, yield and deoxynivalenol (DON) content in grains of wheat following artificial inoculation with Fusarium culmorum/Einfluß von Tebuconazol (Folicur ${ }^{\circledR}$ ) und Prochloraz (Sportale $\left.®\right)$ Behandlungen auf die Entwicklung der Ährenfusariose, den Ertrag und Deoxynivalenol (DON) Gehalt im Korn von Weizen nach künstlicher Inokulation mit Fusarium culmorum. Zeitschrift für Pflanzenkrankheiten und Pflanzenschutz/Journal of Plant diseases and Protection, pp. 33-52.

Miguel, T., Bordini, J. G., Saito, G. H., Andrade, C. G., Ono, M. A., Hirooka, E. Y., Vizoni, É., \& Ono, E. Y. (2015). Effect of fungicide on Fusarium verticillioides mycelial morphology and fumonisin B1 production. Brazilian Journal of Microbiology, 46, 293-299.

Nguyen, L. T., Schmidt, H. A., von Haeseler, A., \& Minh, B. Q. (2014). Iq-tree: a fast and effective stochastic algorithm for estimating maximum-likelihood phylogenies. Molecular Biology and Evolution, 32, 268-274.

Ochiai, N., Tokai, T., Takahashi-Ando, N., Fujimura, M., \& Kimura, M. (2007). Genetically engineered Fusarium as a tool to evaluate the effects of environmental factors on initiation of trichothecene biosynthesis. FEMS Microbiology Letters, 275, 53-61.

Omrane, S., Sghyer, H., Audéon, C., Lanen, C., Duplaix, C., Walker, A. S., \& Fillinger, S. (2015). Fungicide efflux and the MgMFS1 transporter contribute to the multidrug resistance phenotype in Zymoseptoria tritici field isolates. Environmental Microbiology, 17, 2805-2823.

Owczarzy, R., Moreira, B. G., You, Y., Behlke, M. A., \& Walder, J. A. (2008). Predicting stability of DNA duplexes in solutions containing magnesium and monovalent cations. Biochemistry, 47, 5336-5353.

Popiel, D., Koczyk, G., Dawidziuk, A., Gromadzka, K., Blaszczyk, L., \& Chelkowski, J. (2014). Zearalenone lactonohydrolase activity in Hypocreales and its evolutionary relationships within the epoxide hydrolase subset of $\mathrm{a} / \mathrm{b}$ hydrolases. BMC Microbiology, 14, 82.

Price, C. L., Parker, J. E., Warrilow, A. G., Kelly, D. E., \& Kelly, S. L. (2015). Azole fungicides-understanding resistance mechanisms in agricultural fungal pathogens. Pest Management Science, 71, 1054-1058. 
Rogers, B., Decottignies, A., Kolaczkowski, M., Carvajal, E., Balzi, E., \& Goffeau, A. (2001). The pleitropic drug ABC transporters from Saccharomyces cerevisiae. Journal of Molecular Microbiology and Biotechnology, 3, 207-214.

Rozen, S., \& Skaletsky, H. (2000). Primer3 on the WWW for general users and for biologist programmers. Bioinformatics Methods and Protocols, 365-386.

Sá-Correia, I., \& Tenreiro, S. (2002). The multidrug resistance transporters of the major facilitator superfamily, 6 years after disclosure of Saccharomyces cerevisiae genome sequence. Journal of Biotechnology, 98, 215-226.

Saier, M. H. (1999). A functional-phylogenetic system for the classification of transport proteins. Journal of Cellular Biochemistry, 75, 84-94.

Saier, M. H., Tran, C. V., \& Barabote, R. D. (2006). TCDB: the transporter classification database for membrane transport protein analyses and information. Nucleic Acids Research, 34, D181-D186.

Sasnauskas, K., Jomantienė, R., Lebedienè, E., Lebedys, J., Januska, A., \& Janulaitis, A. (1992). Cloning and sequence analysis of a Candida maltosa gene which confers resistance to cycloheximide. Gene, 116, 105-108.

Schmidt-Heydt, M., Baxter, E., Geisen, R., \& Magan, N. (2007). Physiological relationship between food preservatives, environmental factors, ochratoxin and otapksPV gene expression by Penicillium verrucosum. International Journal of Food Microbiology, 119, 277-283.

Schmidt-Heydt, M., Stoll, D., \& Geisen, R. (2013). Fungicides effectively used for growth inhibition of several fungi could induce mycotoxin biosynthesis in toxigenic species. International Journal of Food Microbiology, 166, 407-12.

Simpson, D. R., Weston, G. E., Turner, J. A., Jennings, P., \& Nicholson, P. (2001). Differential control of head blight pathogens of wheat by fungicides and consequences for mycotoxin contamination of grain. European Journal of Plant Pathology, 107, 421-431.

Talas, F., Kalih, R., Miedaner, T., \& McDonald, B. A. (2016). Genome-wide association study identifies novel candidate genes for aggressiveness, deoxynivalenol production, and azole sensitivity in natural field populations of Fusarium graminearum. Molecular Plant-Microbe Interactions, 29, 417-430.

Thurau, T., Beyer, M., Blanck, T., \& Liu, X. (2013). Transcriptional changes of putative Fusarium graminearum transporter sequences in response to trifloxystrobin and deoxynivalenol. Journal of Plant Pathology, 95, S1.29S1.37.

Vermeulen, T., Schoonbeek, H., \& De Waard, M. A. (2001). The $\mathrm{ABC}$ transporter BcatrB from Botrytis cinerea is a determinant of the activity of the phenylpyrrole fungicide fludioxonil. Pest Management Science, 57, 393-402.

Yoshida, M., Nakajima, T., Tomimura, K., Suzuki, F., Arai, M., \& Miyasaka, A. (2012). Effect of the timing of fungicide application on Fusarium head blight and mycotoxin contamination in wheat. Plant Disease, 96, 845-851.

Zhang, Y. J., Fan, P. S., Zhang, X., Chen, C. J., \& Zhou, M. G. (2009a). Quantification of Fusarium graminearum in harvested grain by real-time polymerase chain reaction to assess efficacies of fungicides on fusarium head blight, deoxynivalenol contamination, and yield of winter wheat. Phytopathology, 99, 95-100.

Zhang, Y. J., Yu, J. J., Zhang, Y. N., Zhang, X., Cheng, C. J., Wang, J. X., Hollomon, D. W., Fan, P. S., \& Zhou, M. G. (2009b). Effect of carbendazim resistance on trichothecene production and aggressiveness of Fusarium graminearum. Molecular Plant-Microbe Interactions, 22, 1143-1150. 